\title{
Latency-Optimized Parallelization of the FMM Near-Field Computations
}

\author{
Ivo Kabadshow ${ }^{1}$ and Bruno Lang $^{2}$ \\ 1 John von Neumann Institute for Computing, \\ Central Institute for Applied Mathematics, Research Centre Jülich, Germany \\ i.kabadshow@fz-juelich.de \\ ${ }^{2}$ Applied Computer Science and Scientific Computing Group, \\ Department of Mathematics, University of Wuppertal, Germany
}

\begin{abstract}
In this paper we present a new parallelization scheme for the FMM near-field. The parallelization is based on the Global Arrays Toolkit and uses one-sided communication with overlapping. It employs a purely static load-balancing approach to minimize the number of communication steps and benefits from a maximum utilization of data locality. In contrast to other implementations the communication is initiated by the process owning the data via a put call, not the process receiving the data (via a get call).
\end{abstract}

\section{Introduction}

The simulation of particle systems is a central problem in computational physics. If the interaction between these particles is described using an electrostatic or gravitational potential $\sim 1 / r$, the accurate solution poses several problems. A straightforward computation of all pairwise interactions has the complexity $\mathcal{O}\left(N^{2}\right)$. The Fast Multipole Method (FMM) developed by Greengard and Rokhlin [1] reduces the complexity to $\mathcal{O}(N)$. A detailed depiction of the FMM would be beyond the scope of this paper and can be found elsewhere [2. We will only outline the most important details for the parallelization.

The FMM proceeds in five passes.

- Sort all particles into boxes.

- Pass 1: Calculation and shifting of multipole moments.

- Pass 2: Transforming multipole moments.

- Pass 3: Shifting Taylor-like coefficients.

- Pass 4: Calculation of the far-field energy.

- Pass 5: Calculation of the near-field energy.

The most time-consuming parts of the algorithm are Pass 2 and Pass 5, each contributing approximately $45 \%$ to the overall computing time. Since we have different calculation schemes for Pass 2 and 5, different parallelization schemes are necessary. This paper deals with Pass 5 only, the direct (near-field) pairwise interaction. The sequential version of the near-field computation involves the following steps. 
- Create the particle-box relation ibox in skip-vector form (see Sec. 3.1).

- Calculate all interactions of particles contained in the same box $i$ (routine pass5inbox).

- Calculate all interactions of particles contained in different boxes $i$ and $j$ (routine pass5bibj).

\section{Constraints for the Parallelization}

In order to match the parallelization of the remaining FMM passes and to achieve reasonable efficiency, the following constraints had to be met.

1. Use the Global Arrays Toolkit (GA) for storing global data to preserve the global view of the data.

GA provides a parallel programming model with a global view of data structures. GA uses the ARMCI communication library offering one-sided and non-blocking communication. A detailed description of all GA features can be found in Ref. 4. ARMCI is available on a wide range of architectures. Additionally GA comes with its own memory allocator (MA). To use the available memory efficiently, our implementation will allocate all large local scratch arrays dynamically by MA routines instead of direct Fortran allocation.

2. Minimize the number of communication steps.

All tests were performed on the Jülich Multi Processor (JUMP); a SMP cluster with 41 nodes with 32 IBM Power $4+$ processors and 128 GB RAM per node. The nodes are connected through a high performance switch with peak bandwidth of $1.4 \mathrm{~GB} / \mathrm{s}$ and measured average latencies of $\approx 30 \mu \mathrm{s}$. Shared memory can be accessed inside a node at the cost of $\approx 3 \mu \mathrm{s}$.

Ignoring the latency issue on such machines can have a dramatic impact on the efficiency; see Fig. 1

3. To reduce the communication costs further, try to overlap communication and calculation and use one-sided communication to receive (ga_get) and send (ga_put) data.

\section{Implementation}

In this section we describe some details of the parallel implementation. Related work and other implementation schemes can be found elsewhere 7819 .

\subsection{Initial Data Distribution}

The most memory consuming data arrays are the Cartesian coordinates (xyz), the charges (q), the particle-box relation stored in the ibox vector, and two output arrays storing the data for the potential (fmmpot) and the gradient (fmmgrad). These arrays have to be distributed to prevent redundancy.

The ibox vector is a special auxiliary data structure mapping all charges to the corresponding boxes. To enable fast access in a box-wise search this structure is stored in a skip-vector form (see Fig 2). 


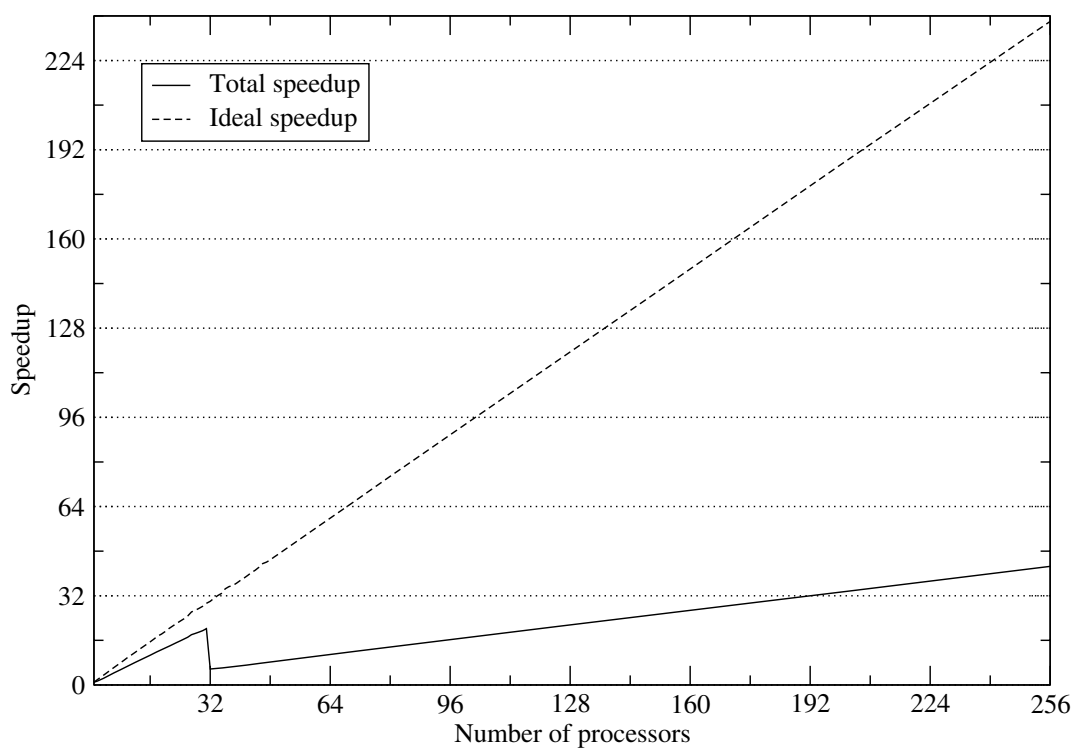

Fig. 1. This figure highlights the latency bottleneck. The speedup for a system with $8^{7} \approx 2 \cdot 10^{6}$ homogeneously distributed particles is shown. Communication is implemented using blocking communication. Each box is fetched separately. The latency switching from $3 \mu \mathrm{s}$ to $30 \mu \mathrm{s}$ can be clearly seen at 32 processors.

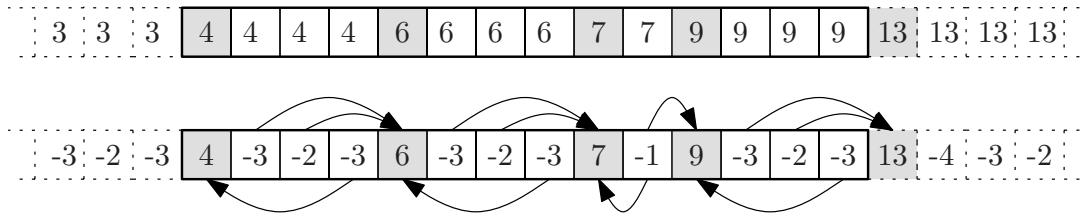

Fig. 2. The diagram illustrates the box management. Empty boxes $(5,8,10,11,12)$ are not stored. The ibox vector associates the box number with each particle. To allow fast access to neighboring boxes the ibox vector is modified into a skipped form. Only the first particle in a box holds the box number, all subsequent charges link forward to the first particle of the next box. Finally, the last particle in a box links back to the first particle of the same box.

\subsection{Design Steps}

The parallel algorithm can be divided into 5 steps:

- Align boxes on processors

- Prepare and initiate communication

- Compute local contributions 
- Compute remote contributions

- Further communication and computation steps, if small communication buffers make them necessary.

Step 1 - Box Alignment. In Passes 1 to 4 of the FMM scheme the particles of some boxes may be stored in more than one processor. These boxes have to be aligned to only one processor. While this guarantees that the Pass 5 subroutine pass5inbox can operate locally, it may introduce load imbalance. However, assuming a homogeneous particle distribution, the load-imbalance will be very small, since the workload for one single box is very small.

The alignment can be done as follows:

- Compute size and indices of the "leftmost" and "rightmost" boxes (w.r.t. Morton ordering; cf. Fig. 3) and store this information in a global array of size $\mathcal{O}$ (nprocs).

- Gather this array into a local array boxinfo containing information from each processor.

- Assign every box such that the processor owning the larger part gets the whole box.

- Reshape the irregular global arrays ibox, xyz, q, fmmgrad, fmmpot.

- Update boxinfo.

After alignment the pass5inbox subroutine can be called locally.
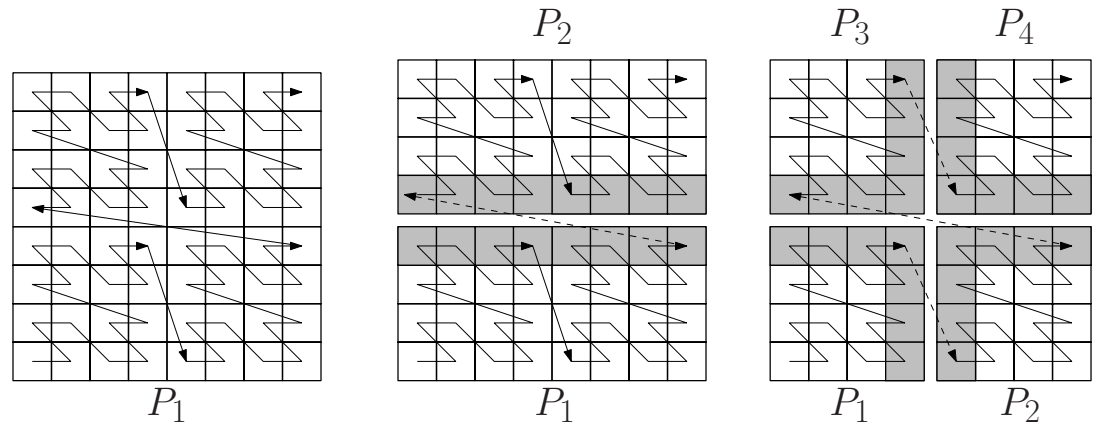

Fig. 3. The data is stored along a Morton-ordered three-dimensional space filling curve (SFC) 6]. The diagram shows a 2D Morton-ordered SFC for the sequential version and a two and four processor parallel version. Since the data is distributed homogeneously the particle/box split is exactly as shown here.

Step 2 - Prepare and Initiate Communication. Like in the sequential program, each processor determines the neighbors of the boxes it holds, i.e. the boxes which lie geometrically above, to the right or in front of the respective box. All other boxes do not need to be considered, since these interactions were already taken into account earlier. This is a special feature of the Morton-ordered 
data structure. Boxes not available locally will be sent by the owner of the box to assure a minimal amount of communication steps. The remote boxes are stored in a local buffer. If the buffer is too small to hold all neighboring boxes, all considered interactions will be postponed until the buffer can be reused.

In order to achieve the $\mathcal{O}$ (nprocs) latency, foreign boxes are put by the processor that holds them into the local memory of the processor requiring them. Each processor has to perform the following steps:

- Determine local boxes that are needed by another processor.

- Determine the processors they reside on.

- Compute the total number of 'items' that every processor will receive.

- Check local buffer space against total size.

- If possible, create a sufficiently large send buffer.

- Issue a ga_put () command to initiate the data transfer.

Step 3 - Compute Local Contributions. Now, that the communication is started, the local portion of the data can be used for computation. All in-box and box-box interactions that are available locally are computed. All computations involving remote/buffered boxes are deferred to Steps $4 / 5$. Step 3 comprises the the calculations of the total Coulomb energy $E_{c}$, the Coulomb forces $\boldsymbol{F}_{c}\left(\boldsymbol{r}_{k}\right)$ and the Coulomb potential $\phi_{c}\left(\boldsymbol{r}_{k}\right)$. Let $n_{i}$ denote the number of particles in box $i$, let $(i, k)$ be the $k$ th particle in box $i$, and let $q_{i, k}$ and $\boldsymbol{r}_{i, k}$ be its charge and position, respectively. Then the total Coulomb energy between all particles in boxes $i$ and $j$, the total Coulomb forces of all particles in box $j$ to a certain particle $(i, k)$ in box $i$, and the corresponding Coulomb potential are given by

$$
\begin{aligned}
E_{c} & =\frac{1}{2} \sum_{k=1}^{n_{i}} \sum_{l=1}^{n_{j}} \frac{q_{i, k} q_{j, l}}{\left|\boldsymbol{r}_{i, k}-\boldsymbol{r}_{j, l}\right|} \\
\boldsymbol{F}_{c}\left(\boldsymbol{r}_{i, k}\right) & =q_{i, k} \sum_{l=1}^{n_{j}} \frac{q_{j, l}}{\left(\boldsymbol{r}_{i, k}-\boldsymbol{r}_{j, l}\right)^{3}}\left(\boldsymbol{r}_{i, k}-\boldsymbol{r}_{j, l}\right) \\
\phi_{c}\left(\boldsymbol{r}_{i, k}\right) & =\sum_{l=1}^{n_{j}} \frac{q_{j, l}}{\left|\boldsymbol{r}_{i, k}-\boldsymbol{r}_{j, l}\right|}
\end{aligned}
$$

respectively. Note that for the in-box case, $i=j$, the terms corresponding to the interaction of a particle with itself must be dropped.

Step 4/5 - Compute Remote Contributions. Since the communication request to fill the buffer was issued before the start of step 3, all communication should be finished by now. After a global synchronization call ga_sync, the buffer is finally used to calculate interactions with the remote/buffered boxes.

\section{Results}

Three different test cases were studied. All test cases contain equally distributed particles (each box contains 8 particles). This guarantees that all boxes are occupied with particles and therefore all possible communication at the processors' 


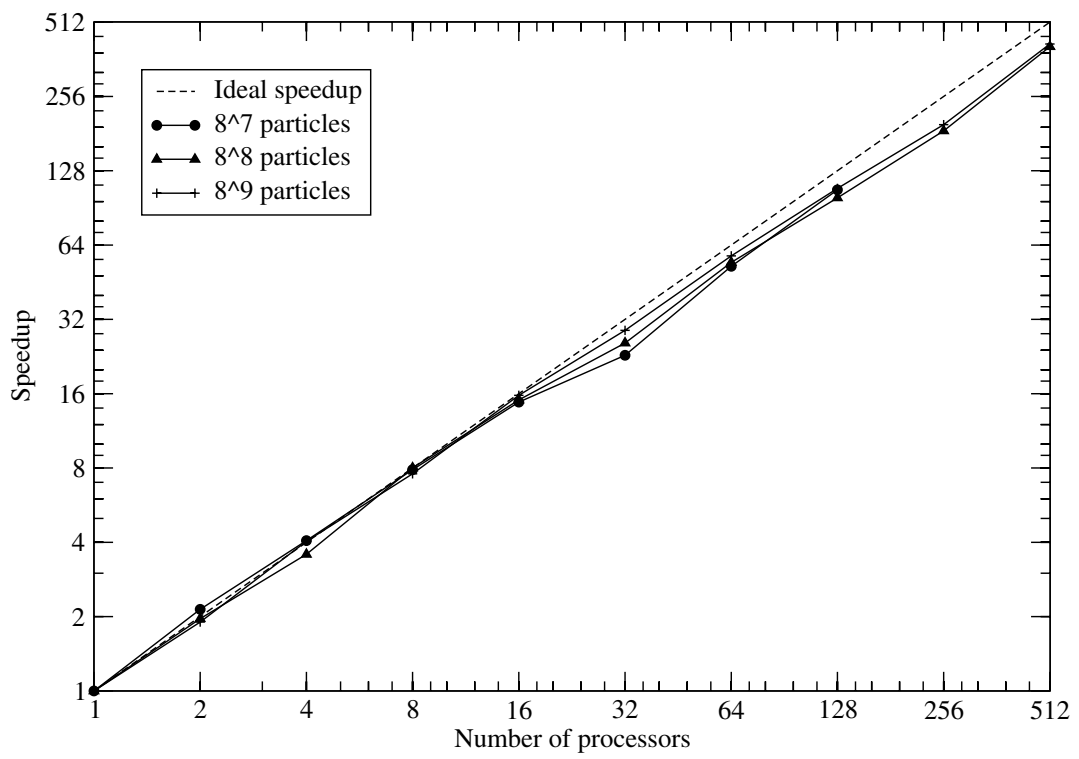

Fig. 4. Three different test cases were computed. The speedup is almost independent from the number of particles even for larger processor numbers. Thus, especially the smallest test case with $8^{7}$ particles benefits from the reduced communication steps.

border will indeed take place. This represents a worst case communication pattern for any number of processors since empty boxes would not be communicated over the network.

The key features can be summarized as follows: All expensive operations (box search, calculation) are done locally. Especially gathering all needed data for each processor is done by the processor owning the data (ga_put), not by the processor receiving the data (ga_get). This saves unnecessary communication time and thus latency. Even for small test cases with few particles per processor the communication can be hidden behind the calculation. The static load-balancing approach outperforms the dynamic approach especially for clusters with long latency times. Compared to the model described in Sec. 1 this approach scales well beyond 32 processors. There is no visible break in the speedup at 32 processors.

\section{Outlook}

The presented scheme assumes an approximately homogeneous distribution of the particles, but can be extended to handle inhomogeneous distributions by introducing "splitting boxes", i.e., dividing large boxes further until a certain box granularity is reached; hence, the workload can again be distributed equally over all processors. 
Table 1. Computation times in seconds/speedups for three different particle systems. For $8^{7}$ particles calculation was done with processor numbers up to 128 , since the total computation time was already below 0.25 seconds and hence results with more processors would suffer from the low measurement precision. The upper limit of 512 processors was due to limitations in our GA implementation.

\begin{tabular}{|c|c|c|c|c|c|c|}
\hline \multirow{2}{*}{$\frac{\text { procs }}{1}$} & \multicolumn{2}{|c|}{$8^{7}$ particles } & \multicolumn{2}{|c|}{$8^{8}$ particles } & \multicolumn{2}{|c|}{$8^{9}$ particles } \\
\hline & 26.78 & - & 211.74 & - & 1654.13 & - \\
\hline 2 & 12.51 & 2.14 & 107.92 & 1.96 & 869.53 & 1.90 \\
\hline 4 & 6.43 & 4.16 & 59.19 & 3.58 & 409.70 & 4.04 \\
\hline 8 & 3.33 & 8.04 & 26.44 & 8.01 & 218.22 & 7.58 \\
\hline 16 & 1.76 & 15.22 & 14.03 & 15.09 & 105.01 & 15.75 \\
\hline 32 & 1.11 & 24.13 & 8.25 & 25.67 & 57.26 & 28.89 \\
\hline 64 & 0.45 & 59.51 & 3.89 & 54.43 & 28.59 & 57.86 \\
\hline 128 & 0.21 & 127.52 & 2.13 & 99.41 & 15.23 & 108.61 \\
\hline 256 & - & - & 1.14 & 185.74 & 8.41 & 196.69 \\
\hline 512 & — & - & 0.52 & 407.19 & 3.96 & 417.71 \\
\hline
\end{tabular}

\section{Acknowledgements}

The authors acknowledge the support by H. Dachsel for his sequential FMM code, as well as B. Kuehnel for his work on the parallel code as a guest student at research centre Jülich.

\section{References}

1. L. Greengard and V. Rokhlin: A fast algorithm for particle simulations. J. Comput. Phys. 73, No.2 (1987) 325-348

2. C. A. White and M. Head-Gordon: Derivation and efficient implementation of the fast multipole method. J. Chem. Phys. 101 (1994) 6593-6605

3. H. Dachsel: An error-controlled Fast Multipole Method. (in preparation)

4. J. Nieplocha, B. Palmer, V. Tipparaju, M. Krishnan, H. Trease and E. Apra: Advances, Applications and Performance of the Global Arrays Shared Memory Programming Toolkit. IJHPCA, 20, No. 2, (2006) 203-231

5. J. Nieplocha, V. Tipparaju, M. Krishnan, and D. Panda: High Performance Remote Memory Access Communications: The ARMCI Approach. IJHPCA, 20, No. 2, (2006) 233-253

6. M.F. Mokbel, W.G. Aref and I. Kamel: Analysis of multi-dimensional space-filling curves. Geoinformatica 7, No.3 (2003) 179-209

7. L. Greengard and W.D. Gopp: A parallel version of the multipole method. Computers Math. Applic. 20, No. 7 (1990) 63-71

8. J. Kurzak and B.M. Pettitt: Communications overlapping in fast multipole particle dynamics methods. J. Comput. Phys. 203 (2005) 731-743

9. J. Kurzak and B.M. Pettitt: Massively parallel implementation of a fast multipole method for distributed memory machines. J. Par. Dist. Comp. 65 (2005) 870-881 